\title{
The LOFAR view of FR 0 radio galaxies
}

\author{
A. Capetti ${ }^{1}$, M. Brienza ${ }^{2,3}$, R. D. Baldi ${ }^{4,5,6}$, G. Giovannini ${ }^{2,3}$, R. Morganti ${ }^{7,8}$, M. J. Hardcastle ${ }^{9}$, \\ H. J. A. Rottgering ${ }^{10}$, G. F. Brunetti ${ }^{3}$, P. N. Best ${ }^{11}$, and G. Miley ${ }^{10}$
}

1 INAF - Osservatorio Astrofisico di Torino, Strada Osservatorio 20, 10025 Pino Torinese, Italy e-mail: capetti@oato.inaf.it

2 Dipartimento di Fisica e Astronomia, Università di Bologna, Via P. Gobetti 93/2, 40129 Bologna, Italy

3 INAF - Istituto di Radio Astronomia, Via P. Gobetti 101, 40129 Bologna, Italy

4 Department of Physics \& Astronomy, University of Southampton, Hampshire, SO17 1BJ Southampton, UK

5 Dipartimento di Fisica, Universitá degli Studi di Torino, via Pietro Giuria 1, 10125 Torino, Italy

6 INAF - Istituto di Astrofisica e Planetologia Spaziali, via Fosso del Cavaliere 100, 00133 Roma, Italy

7 ASTRON, the Netherlands Institute of Radio Astronomy, Postbus 2, 7990 AA Dwingeloo, The Netherlands

8 Kapteyn Astronomical Institute, University of Groningen, PO Box 800, 9700 AV Groningen, The Netherlands

9 Centre for Astrophysics Research, University of Hertfordshire, College Lane, Hatfield AL10 9AB, UK

${ }^{10}$ Leiden Observatory, Leiden University, PO Box 9513, 2300 RA Leiden, The Netherlands

11 SUPA, Institute for Astronomy, Royal Observatory, Blackford Hill, Edinburgh EH9 3HJ, UK

Received 16 June 2020 / Accepted 14 August 2020

\begin{abstract}
We explore the low-frequency radio properties of the sources in the Fanaroff-Riley class 0 catalog (FR0CAT) as seen by the LowFrequency ARray (LOFAR) observations at $150 \mathrm{MHz}$. This sample includes 104 compact radio active galactic nuclei (AGN) associated with nearby $(z<0.05)$ massive early-type galaxies. Sixty-six FR0CAT sources are in the sky regions observed by LOFAR and all of them are detected, usually showing point-like structures with sizes of $\lesssim 3-6 \mathrm{kpc}$. However, $12 \mathrm{FR} 0$ s present resolved emission of low surface brightness, which contributes between $5 \%$ and $40 \%$ of the total radio power at $150 \mathrm{MHz}$, usually with a jetted morphology extending between 15 and $50 \mathrm{kpc}$. No extended emission is detected around the other FR $0 \mathrm{~s}$, with a typical luminosity limit of $\lesssim 5 \times 10^{22} \mathrm{~W} \mathrm{~Hz}^{-1}$ over an area of $100 \mathrm{kpc} \times 100 \mathrm{kpc}$. The spectral slopes of FR 0 s between $150 \mathrm{MHz}$ and $1.4 \mathrm{GHz}$ span a broad range $(-0.7 \lesssim \alpha \lesssim 0.8)$ with a median value of $\bar{\alpha} \sim 0.1 ; 20 \%$ of them have a steep spectrum $(\alpha \gtrsim 0.5)$, which is an indication of the presence of substantial extended emission confined within the spatial resolution limit. The fraction of FR 0s showing evidence for the presence of jets, by including both spectral and morphological information, is at least $\sim 40 \%$. This study confirms that FR 0s and FR Is can be interpreted as two extremes of a continuous population of jetted sources, with the FR 0s representing the low end in size and radio power.
\end{abstract}

Key words. galaxies: active - galaxies: jets - galaxies: elliptical and lenticular, cD

\section{Introduction}

The majority of the radio-active galactic nuclei associated with low redshift galaxies detected in recent surveys at $1.4 \mathrm{GHz}$ (for example, Best \& Heckman 2012) are compact, with corresponding linear sizes $\lesssim 10$ kiloparsecs (Baldi \& Capetti 2009). Earlier surveys (performed at a lower frequency and sensitivity) were instead dominated by sources extending over scales of hundreds of kpc (see Hardcastle et al. 1998). The "compact" sources were named "FR 0s" (Ghisellini et al. 2011; Sadler et al. 2014; Baldi et al. 2015) as a convenient way to include them into the canonical Fanaroff \& Riley (1974) classification scheme of radio galaxies (RGs), referring to their lack of extended radio emission. The information available from observations of FR 0 s is generally very limited, even in the radio band. It is then still unclear as to the nature of these sources and how they are related to the other classes of RGs.

Baldi et al. (2018a) selected a sample of compact radio sources named FROCAT in order to perform a systematic study of FR 0s. FR0CAT is formed by compact radio sources with a redshift $\leq 0.05$ selected by combining observations from the National Radio Astronomy Observatory Very Large Array Sky
Survey (NVSS; Condon et al. 1998), the Faint Images of the Radio Sky at Twenty centimeters survey, (FIRST, Becker et al. 1995; Helfand et al. 2015), and the Sloan Digital Sky Survey (SDSS; York et al. 2000). The FR0CAT selection is limited to the galaxies in which various methods (see Best \& Heckman 2012 for details) indicate that the emission is due to a radioAGN, thus excluding star-forming galaxies. Baldi et al. (2018a) included the sources that are brighter than $5 \mathrm{mJy}$ and with a limit to their angular size of 4 " in the FIRST images for a total of 104 sources. Their luminosities at $1.4 \mathrm{GHz}$ are in the range $10^{22} \lesssim L_{1.4 \mathrm{GHz}} \lesssim 10^{24} \mathrm{~W} \mathrm{~Hz}^{-1}$.

The key question about the FR 0s pertains to why they do not show the extended radio emission that characterizes the other classes of RGs: and, additionally, whether this is due to different properties of their central engines. Are FR 0s not able to produce relativistic jets or is there an evolutionary link between them, that is to say, whether FR Os are an early stage of FR Is.

While the radio structure of FR $0 \mathrm{~s}$ and FR Is is different, the nuclear and host galaxy properties of these two classes are very similar (Baldi et al. 2018a; Torresi et al. 2018). There is only a difference in the host optical luminosity of the two classes, those of the FR Is being $\sim 60 \%$ brighter that 
those of the FR 0s, but with a large superposition of the two distributions.

Based on the relative number density of the two classes Baldi et al. (2018a) were able to discard the scenario in which all FR 0s are young RGs which will eventually evolve into extended radio sources. Nonetheless, there must be an intermediate evolutionary stage between the initial nuclear activity of FR Is and FR IIs before they reach their typical linear sizes of hundreds of kpc: some FR 0s are expected to be small because they are young. Capetti et al. (2019) estimated that the fraction of FR 0s with a high curvature convex spectrum, typical of young radio galaxies, is at most $\sim 15 \%$.

FR 0s might instead be recurrent sources, characterized by short phases of activity. Alternatively, Baldi et al. (2015) suggested that what distinguished FR Os from FR Is is the lowerbulk Lorentz factor of the jets in FR 0s, which are disrupted before they can emerge from the host galaxies. Constraints on the jet speed and structure in FR 0s might be obtained from high resolution imaging. The very long baseline observations analyzed by Cheng \& An (2018) suggest the presence of jetted structure in all but one of the sources of the subsample of 14 bright (flux densities $>50 \mathrm{mJy}$ ) FR 0 s they studied. Their pc-scale structures indicate the presence of a broad range of relativistic beaming factors.

Capetti et al. (2020) explored the large-scale environment of FR 0s. They found that FR Os are located in regions with an average number of galaxies that is lower by a factor two with respect to FR I. This difference is driven by the large fraction $(63 \%)$ of FR 0s that are located in groups formed of fewer than 15 galaxies. FR Is rarely (17\%) inhabit an environment like this. One possibility to account for the connection between environment and the properties of the extended radio emission is related to the stronger adiabatic losses of the radio-emitting plasma (for example, Longair 1994) in the poorer environment of the FR 0s. However, Baldi et al. (2019) showed that most FR 0s are still confined well within the core of the hot corona of their host and the similarity of FR 0 and FR I host galaxies suggests that their coronae also have similar distribution of hot gas. The differences in environment between FR 0s and FR Is can instead be due to an evolutionary link between local galaxy density, black hole spin (Garofalo \& Singh 2019), jet power, and extended radio emission. In addition to the lack of substantial extended radio emission that defines the FR 0 class, the properties of the large scale environment represent the first significant difference between these two populations of low-power radio galaxies.

In this context, low-frequency and high sensitivity radio observations of FR 0s might be used to address the following questions:

(1) Do FR 0s show low-frequency extended emission? If FR 0s are indeed recurrent sources, one might expect to detect relic emission from a previous activity phase, best observable at $\mathrm{MHz}$-frequencies due to its steep spectrum.

(2) What is the low-frequency spectral shape of FR 0s? Observations at high resolution, required to spatially isolate any small-scale extended emission, are only available for a minority of FR 0s. The spectral index information can be used to infer the fraction of optically thin, hence extended, emission present in FR 0s overcoming the limited spatial resolution. The large frequency leverage opened by the LOFAR (van Haarlem et al. 2013) images is optimally suited to perform this study.

We already studied the low-frequency properties of FR 0 radio galaxies (Capetti et al. 2019) by using the Alternative Data Release of the TIFR GMRT Sky Survey (TGSS, GMRT; Swarup et al. 1991; Intema et al. 2017). This analysis was how- ever limited to a flux density limit of $17.5 \mathrm{mJy}$ and it returned an association for only 37 out of 104 FR 0 of the FR0CAT sample. Nonetheless it was possible to conclude that (1) most FR 0s $(92 \%)$ have a flat or inverted spectral shape $(\alpha<0.5)^{1}$ between $150 \mathrm{MHz}$ and $1.4 \mathrm{GHz}$ and (2) no extended emission is detected around them, corresponding to a luminosity limit of $\lesssim 4 \times 10^{23} \mathrm{~W} \mathrm{~Hz}^{-1}$. The observations that are being obtained with LOFAR, thanks to their higher depth and resolution, can be used to improve significantly our knowledge of the low-frequency radio properties of FR 0s.c

The paper is organized as follows: in Sect. 2 we describe the LOFAR observations available for the FROCAT sample. The results are presented in Sect. 3 and discussed in Sect. 4. In Sect. 5 we draw our conclusions.

\section{The LOFAR observations}

The LOFAR Two-metre Sky Survey (LoTSS, Shimwell et al. 2017) will cover the whole northern sky with 3168 pointings of eight hours ${ }^{2}$ of dwell time each in the frequency range between 120 and $168 \mathrm{MHz}$. The LoTSS first data release (DR1; Shimwell et al. 2019) presented the results obtained from observations of 424 square degrees in the HETDEX Spring Field. The final release images were obtained by combining the images from individual pointings of the survey, producing mosaics covering the region of interest at a median sensitivity of $71 \mu \mathrm{Jy}^{\text {beam }^{-1}}$. The flux density scale was adjusted to ensure consistency with previous surveys (see Hardcastle et al. 2016 for further details).

The second LoTSS data release (DR2) will consist of two contiguous fields at high Galactic latitude centered around $0 \mathrm{~h}$ and $13 \mathrm{~h}$ and covering approximatively 5700 square degrees (Shimwell et al. in preparation). The DR2 provides fully calibrated mosaics at a resolution of $\sim 6^{\prime \prime}$, catalogs, and it includes 42 FR 0s (marked as "DR2" in Table A.1). FR 0s images can also be obtained from individual LoTSS pointings, outside the DR2 area. We restrict the analysis to the 24 objects (the "P" sources in Table A.1) with an offset from the field's center smaller than $3^{\circ}$ in order to limit ourselves to the regions of lower noise. The total number of FR 0 s with currently available LOFAR data is then 66, that is, about two thirds of the FR0CAT sample.

We estimated the rms of each image in various regions, usually centered $45^{\prime}$ from the FR 0 s. For the FR 0 s falling into the DR2 the median rms is $85 \mu \mathrm{Jy}_{\text {beam }}{ }^{-1}$, while this is $240 \mu \mathrm{Jy}$ beam $^{-1}$ for the "P" sources.

The photometry of the sources included in the DR2 is available from the internally released catalog, while for the sources included in the "P" group we instead measured their flux density from their LOFAR images. The flux density errors are dominated by the uncertainties in the absolute calibration and are typically $\sim 10 \%$.

The list of FR Os covered by LOFAR images is presented in Table A.1 where we indicate their SDSS name, their redshift, the local rms of the image used, the flux density and luminosity at $150 \mathrm{MHz}$ of the central component from the LOFAR data (for the extended sources we also give the total flux density and a morphological description), the $1.4 \mathrm{GHz}$ flux density from FIRST, and the spectral index between these frequencies. In the last column a code indicates the origin of the image ("DR2" or "P").

\footnotetext{
1 Spectral indices $\alpha$ are defined as $F_{v} \propto v^{-\alpha}$.

2 The low declination fields $\left(\delta \lesssim 20^{\circ}\right.$ ) will instead be observed for 12 hours.
} 


\section{Results}

\subsection{Morphology of FR Os}

All FR0CAT sources with LOFAR observations are detected at $150 \mathrm{MHz}$. Most of them are point-like with a deconvolved major axis $\lesssim 6^{\prime \prime}$ (the median value is $5^{\prime \prime} 0$ ), that is, $4 \mathrm{kpc}$ at their median redshift.

There are 12 clearly extended sources (see Table A.1 and Figs. 1 and 2$)^{3}$. The most commonly observed morphology among them (in five sources, namely, J1025+17, J1044+43, $\mathrm{J} 1116+29, \mathrm{~J} 1134+49$, and $\mathrm{J} 1722+30$ ) is the presence of two rather symmetric jets, usually bent with an S-shape structure, with a total extent between 20 and $40 \mathrm{kpc}$. In three sources (J0916+17, J1541+45, and J1604+17) we see a head-tail structure reaching $\sim 50 \mathrm{kpc}$; in one of them $(\mathrm{J} 1604+17)$ a second source is seen $\sim 30^{\prime \prime}$ north, along the radio tail, associated with a spiral galaxy interacting with the host of the radio source. Two FR0CAT sources (J0807+14 and J1605+14) have instead a core-jet shape, while one $(\mathrm{J} 1703+24)$ is barely resolved and of uncertain morphology. The higher resolution observations of this source at $1.4 \mathrm{GHz}$ presented by Baldi et al. (2019) show a double-lobed structure, with a total extent of $\sim 15 \mathrm{kpc}$. The last object $(\mathrm{J} 0910+18)$ is quite peculiar with a bright central source superposed to a large scale plateau of diffuse radio emission. The low resolution (20") LOFAR image (see Fig. 3) reveals that this plateau is just the central part of an elongated radio structure, extending over $\sim 4^{\prime}$, that is, $\sim 150 \mathrm{kpc}$. In addition, J1308+43 (see Fig. 4) has a low brightness diffuse feature centered $30^{\prime \prime}$ $(\sim 18 \mathrm{kpc})$ to the NNW side, but in this case it is detached from the radio core and not obviously associated with the FR 0 .

For the extended sources we measured both the flux density of the central component and the total flux density, measured integrating in the region included by the $3 \sigma$ isophote. The central component always accounts for a large fraction of the total emission, from $40 \%$ to $93 \%$. The luminosities of the extended emission range from $10^{22}$ to $3 \times 10^{23} \mathrm{~W} \mathrm{~Hz}^{-1}$. These structures were not visible in the TGSS images.

None of the extended structures detected in the LOFAR images of the FR0CAT sources has a counterpart in the FIRST images. This is not surprising if they are steep spectra features $(\alpha=0.7)$ given their brightness levels at $150 \mathrm{MHz}(0.6-$ $1.2 \mathrm{mJy}$ beam $^{-1}$ ) leading to an expected surface brightness of $0.1-0.2 \mathrm{mJy}^{-1}$ beam $^{-1}$ at $1.4 \mathrm{GHz}$. The lower limits to the spectral indices of the extended emission do provide strong constraints, with typical limits $\alpha \gtrsim 0.4-0.6$, except for the head-tail source J0916+17 ( $\alpha \gtrsim 0.9$ ). However, although the LOFAR spatial resolution $\left(\sim 5^{\prime \prime}\right)$ is very similar to that achieved by FIRST, in the latter survey the structures larger than $\sim 1^{\prime}$ might be at least partly resolved out, given the lack of short baselines in the $u-v$ coverage (Helfand et al. 2015). An indication that this is indeed the case comes from the ratio between the NVSS and FIRST flux densities of the $12 \mathrm{FR}$ 0s extended at $150 \mathrm{MHz}$ that, in seven $^{4}$ of them, is $1.30<F_{\text {NVSS }} / F_{\text {FIRST }}<1.45$ : similarly high ratios are measured only in 14/104 FR 0s of the complete sample (Capetti et al. 2017). However, variability, combined with a high core dominance, prevents us from using the NVSS/FIRST flux ratio to extract robust measurements of the spectral slope of the extended emission. In fact, FR 0 s show signs of

\footnotetext{
3 Somewhat surprisingly, the fraction of extended sources is lower $(12 \%)$ in the deeper DR2 observations than in those covered by the shallower single survey's pointings $(29 \%)$.

4 Namely, J0916+17, J1044+43, J1134+49, J1541+45, J1604+17, $\mathrm{J} 1703+24$, and $\mathrm{J} 1722+30$.
}

variability over a timescales of a few years. This conclusion is based on multi-epoch VLBI images (Cheng \& An 2018) and on the presence of a large number of FR 0 s in which the flux density in the FIRST images significantly exceeds that measured from the NVSS (Baldi et al. 2018a). Furthermore, hints of variability are also identified when comparing the 150-MHz LOFAR flux densities with the TGSS flux densities at the same frequency, as shown in Fig. 6.

We estimate the limit on the flux density of any extended emission at $150 \mathrm{MHz}$ around the remaining FR 0s by considering an area of $100 \mathrm{kpc} \times 100 \mathrm{kpc}$, the typical size of the edgedarkened FR I sources (forming the sample named FRICAT, Capetti et al. 2017) selected from the same catalog of RGs from which we extracted the FR 0s. At the median redshift of the FR0CAT of 0.037 , this corresponds to $\sim 135^{\prime \prime} \times 135^{\prime \prime}$. The median rms is $0.15 \mathrm{mJy}^{\text {beam }}{ }^{-1}$ and this leads to an upper limit over this area of $\sim 10 \mathrm{mJy}$, corresponding to a luminosity of $\lesssim 5 \times 10^{22} \mathrm{~W} \mathrm{~Hz}^{-1}$.

\subsection{Radio spectral properties of FR Os}

In Fig. 5 we compare the flux densities of the FR0CAT sources in FIRST and LOFAR. The FR0CAT sources show a rather large spread in spectral indices, ranging from $\alpha \sim-0.7$ to $\sim 0.8$, with a median value of $\bar{\alpha} \sim 0.1$. The fraction of sources with a steep spectrum $(\alpha>0.5)$ is $\sim 20 \%$, a larger value with respect to what we obtained combining TGSS and NVSS data $(\sim 8 \%$, Capetti et al. 2019). The comparison of the spectral indices for the 29 sources observed by LOFAR and detected by TGSS indicate that in the majority of them (25) the difference in spectral slope is smaller than 0.1 , with no systematic offset. In the remaining four sources the difference is larger, reaching $\Delta \alpha=$ 0.4. This effect is likely due to the flux losses in the FIRST and TGSS images, with respect to the NVSS and LOFAR observations, respectively.

The central components associated with the 12 extended sources are all among the upper half of the luminosity distribution of FR $0 \mathrm{~s}$, having a median luminosity of $10^{23} \mathrm{~W} \mathrm{~Hz}^{-1}$, and a median slope of $\bar{\alpha} \sim 0.4$.

\section{Discussion}

The definition of compact radio sources, at the base of our study of FR 0s, clearly depends on the spatial resolution, depth, and frequency of the available images. Baldi et al. (2019) presented high resolution images (between $0^{\prime \prime} .3$ and $1^{\prime \prime}$ ) of a subsample of 18 FR0CAT sources, detecting extended emission (on a scale between 2 and $14 \mathrm{kpc}$ ) only in four of them. The LOFAR observations provide us with additional evidence that the inclusion of a radio source into the FR 0s class is nearly independent of the characteristics of the data used and that, consequently, this class of radio galaxies is rather well defined. In particular, they confirm that FR 0s are in general compact sources, of high core dominance. Even when extended radio emission is detected in the FR 0s, thanks to the deep LOFAR images, it represents a small fraction of the total flux densities in these objects, ranging from $\sim 5$ to $\sim 40 \%$. For the unresolved FR 0 s, we set a limit to the luminosity of any extended emission of $\lesssim 5 \times 10^{22} \mathrm{~W} \mathrm{~Hz}^{-1}$ within an area of $100 \mathrm{kpc} \times 100 \mathrm{kpc}$. As reference, the FRICAT sources in Capetti et al. (2017) have predicted luminosities at $150 \mathrm{MHz}$ (by assuming a spectral slope of 0.7 between 150 and $1400 \mathrm{MHz}$ ) in the range $\sim 10^{24}-10^{26} \mathrm{~W} \mathrm{~Hz}^{-1}$.

The properties of the 12 extended sources in the LOFAR images can be compared with those of the FR Is selected from 
A\&A 642, A107 (2020)
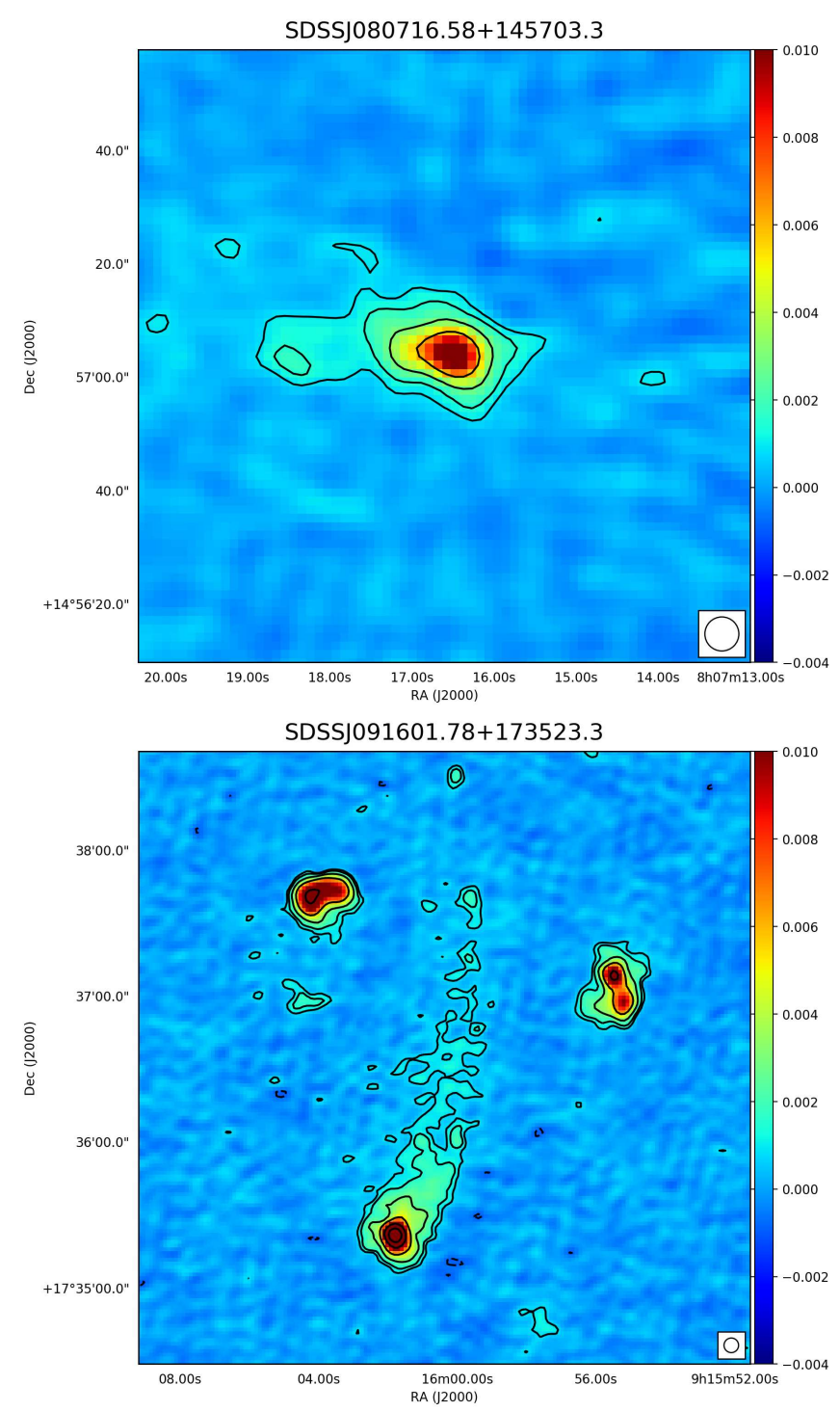

SDSSJ $104403.68+435412.0$

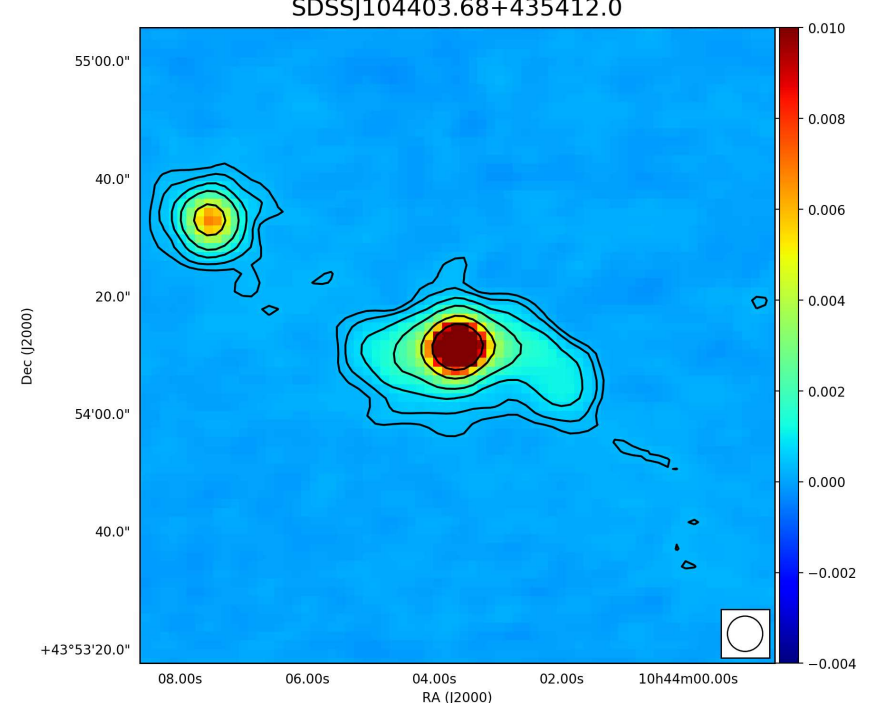

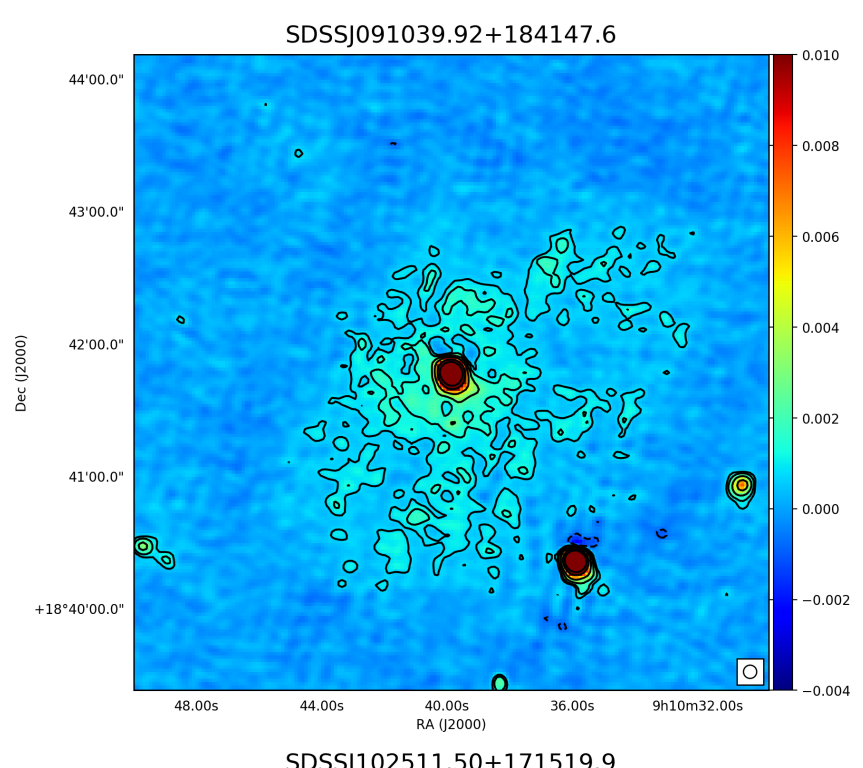
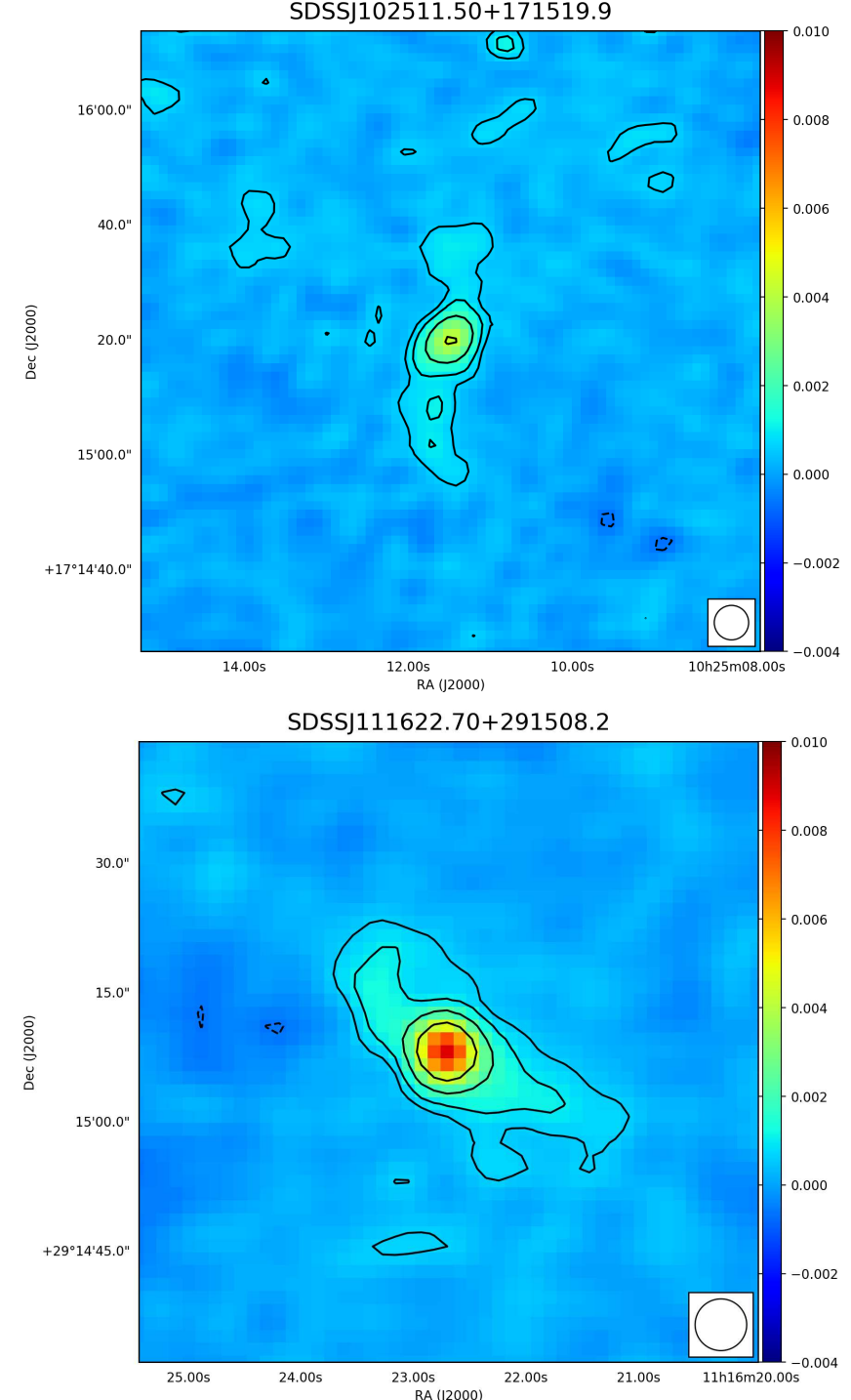

Fig. 1. LOFAR images of the extended FR0CAT sources at a resolution of $\sim 6^{\prime \prime}$. The contour levels follow the sequence $-3,3,5,10,20,50,100 \sigma$, where $\sigma$ is the local rms, as reported in Table A.1, 0.20, 0.20, 0.27, 0.21, 0.09, and $0.20 \mathrm{mJy} \mathrm{beam}^{-1}$, respectively. 
A. Capetti et al.: The LOFAR view of FR 0 radio galaxies
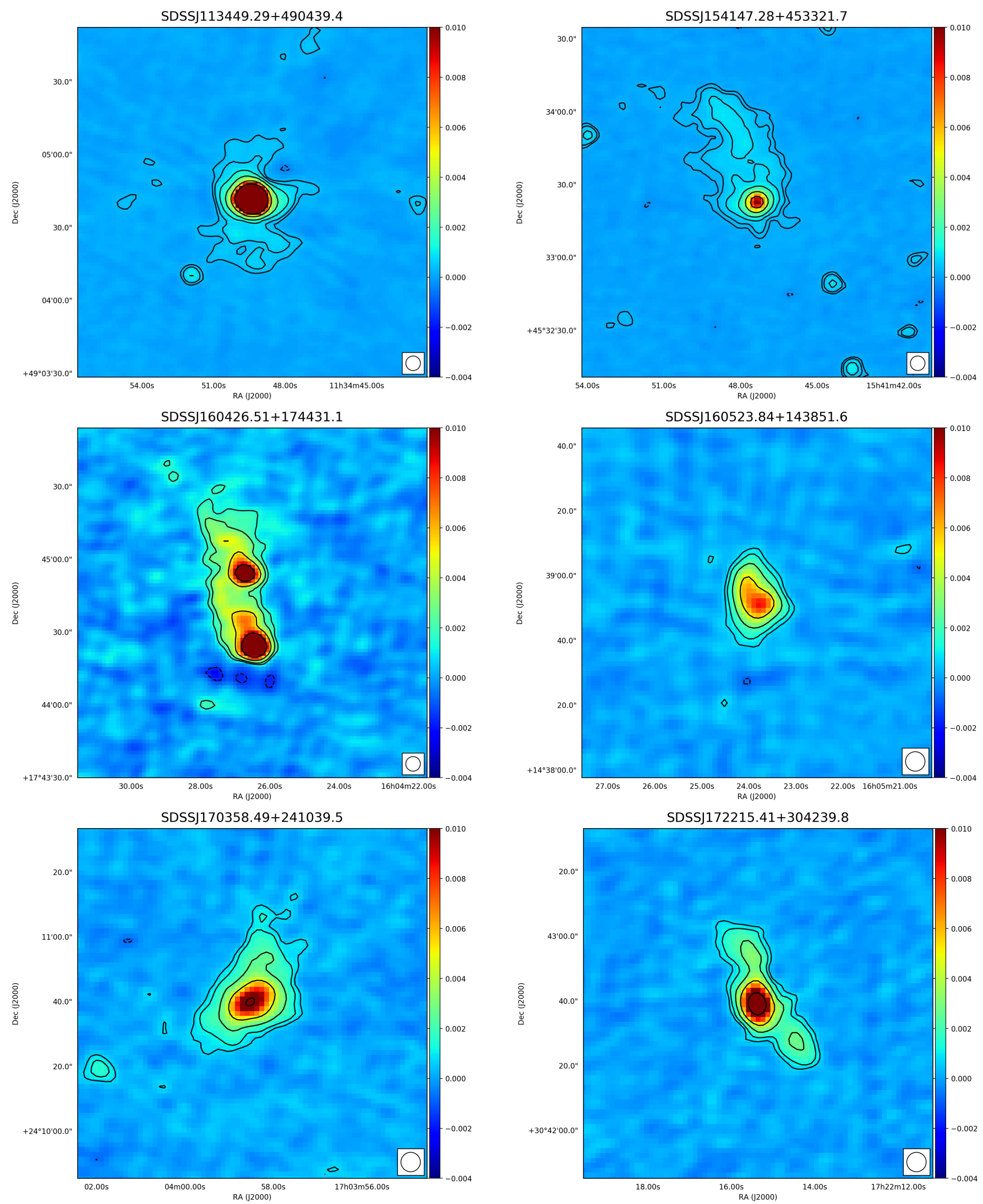

Fig. 2. LOFAR images of the extended FR0CAT sources at a resolution of $\sim 6^{\prime \prime}$. The contour levels follow the sequence $-3,3,5,10,20,50,100 \sigma$, where $\sigma$ is the local $\mathrm{rms}$, as reported in Table A.1, $0.14,0.10,0.38,0.21,0.22$, and $0.10 \mathrm{mJy}$ beam $^{-1}$, respectively. 


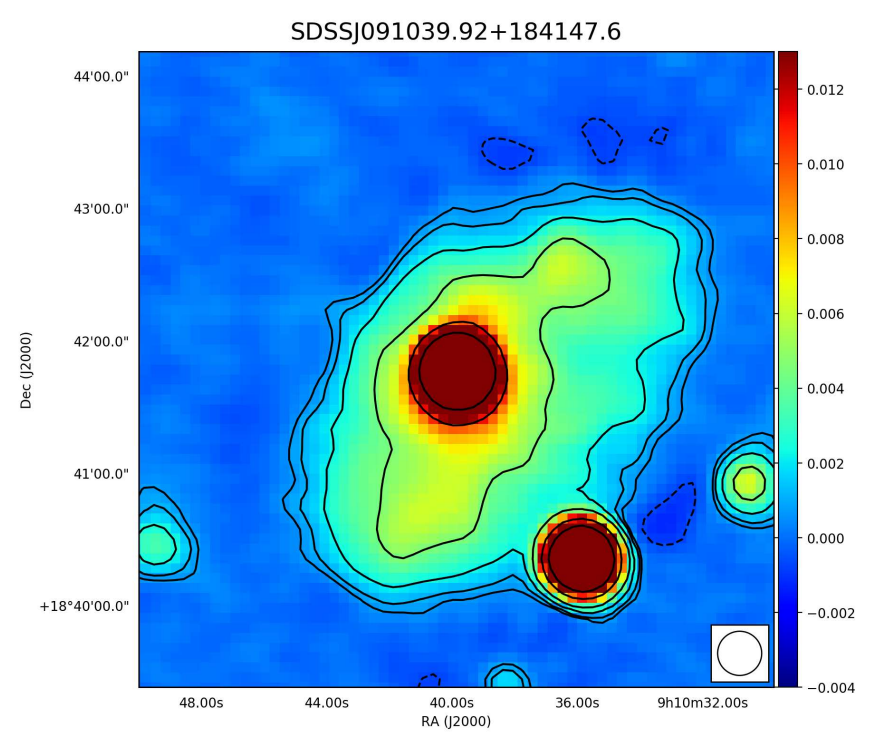

Fig. 3. LOFAR image at low resolution $\left(\sim 20^{\prime \prime}\right)$ of J0910+18, best showing the elongated diffuse emission. The contour levels follow the sequence $-3,3,5,10,20,50,100 \sigma$, where $\sigma=500 \mu \mathrm{Jy} \mathrm{beam}^{-1}$.

the FIRST images (Capetti et al. 2017). Their median optical magnitude is $M_{r}=-22.38$, a value intermediate between that measured for the FR0CAT and FRICAT samples $\left(M_{r}=-22.05\right.$ and $M_{r}=-22.52$, respectively). From the point of view of the environment, the median number of cosmological neighbors ${ }^{5}$ is $N_{\mathrm{cn}}^{2000}=25$ for the extended sources, to be compared to 13 and 44 for the FR0CAT and FRICAT objects, respectively. Conversely, these 12 FR 0s do not differ from the remaining sources from the point of view of optical line properties. This analysis indicates that large scale radio structures are preferentially seen in FR 0s whose host galaxies are more luminous and are located in a denser environment than average. As already mentioned in the Introduction, the radio structure of most FR 0s is confined within the core of their hosts and is not directly affected by the larger scale environment. Nonetheless, the larger structures that a minority of FR 0s are able to form might be easier to detect in a denser environment where their expansion and the adiabatic losses are reduced. The role of host's mass and environment in determining the properties of a radio source is still unclear, but these results strengthen the link between these two parameters with the jet power and the ability of the AGN to produce large scale radio emission.

Almost all of the FR0CAT sources with extended radio emission (detected with either LOFAR or with the VLA) show the typical morphology usually associated with low-power jets: collimated structures, albeit often distorted, of rapidly declining brightness at increasing distance from the nucleus. This result argues against the possibility that these structures are relic emission from previous phases of activity. In such objects the radio emission is generally characterized by a diffuse morphology (see Brienza et al. 2016), due to the lateral expansion that occurs when the jets cease to replenish the large scale structures.

The presence of jets in FR 0s can also be argued, besides their direct morphological detection, from their spectral radio properties. The fraction of sources with a steep spectrum

\footnotetext{
5 "Cosmological neighbors" are defined as the galaxies lying within a projected radius of $2 \mathrm{Mpc}$ and having a spectroscopic redshift $z$ differing by less than 0.005 from the radio galaxy in the center of the field examined.
}

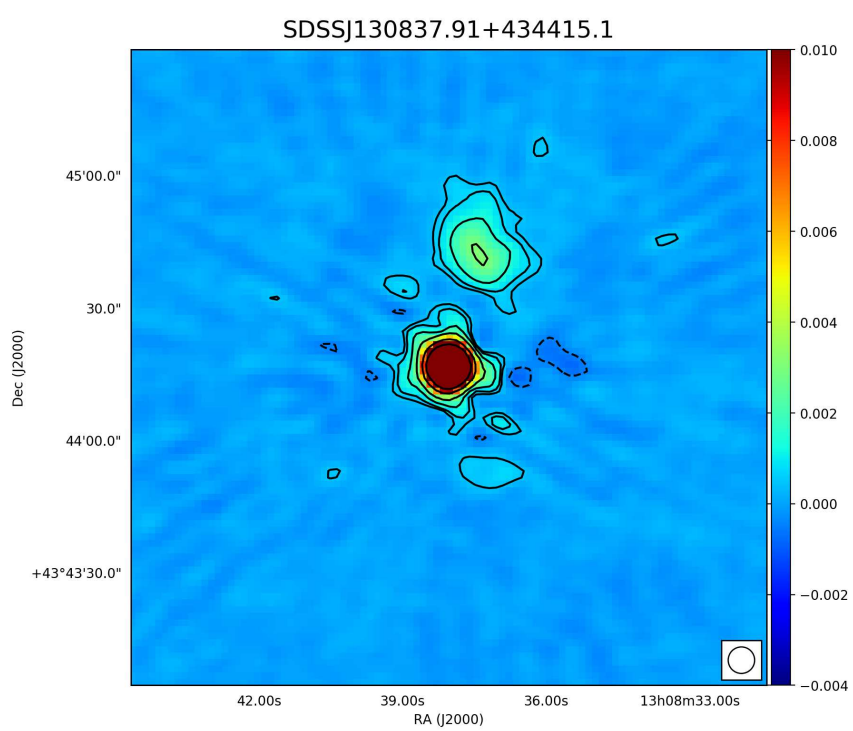

Fig. 4. LOFAR image of $\mathrm{J} 1308+43$ (resolution of $\sim 6^{\prime \prime}$ ) shows $\sim 30^{\prime \prime}$ north of the FR 0 core a detached diffuse radio structure. The contour levels follow the sequence $-3,3,5,10,20,50,100 \sigma$, where $\sigma=0.14$ mJy beam $^{-1}$.

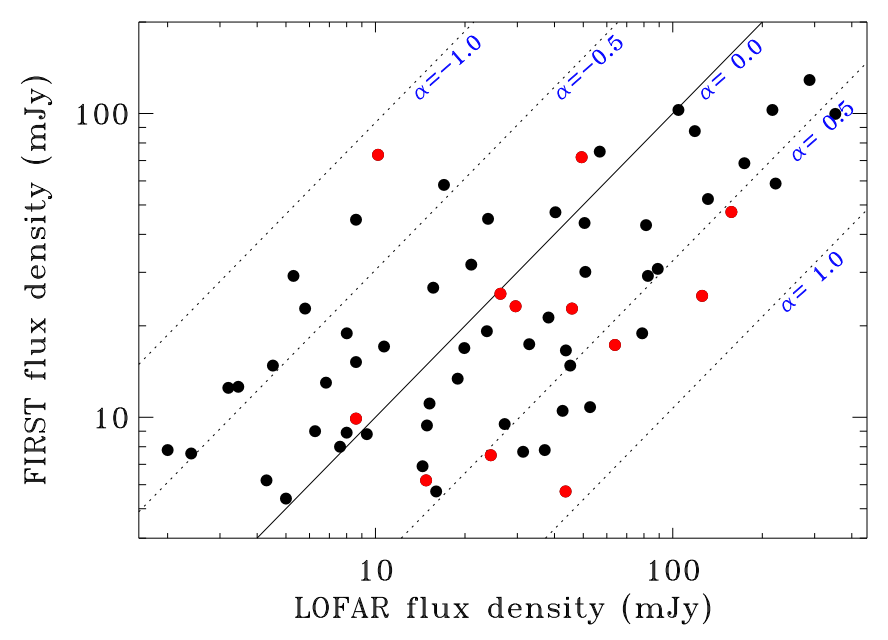

Fig. 5. Comparison of the flux densities of the compact components of the FR0CAT sources in LOFAR (at $150 \mathrm{MHz}$ ) and FIRST (at $1.4 \mathrm{GHz}$ ). The red dots represent the 12 sources extended in the LOFAR images. The lines are the loci of constant spectral indices $\alpha$ (defined as $F_{v} \propto$ $\left.v^{-\alpha}\right)$ at the values indicated.

( $\alpha>0.5$ ) is $\sim 20 \%$, represents an indication of the presence of substantial extended emission confined within the LOFAR spatial resolution limit $(\sim 3-6 \mathrm{kpc})$. This suggestion is confirmed by considering the sources showing extended emission in the high resolution VLA data of Baldi et al. (2019). Three of these four sources have LOFAR observations: J1703+24 is extended in both datasets, while $\mathrm{J} 1213+50$ and $\mathrm{J} 1559+44$ are both pointlike in the LOFAR images. While the first source has a size of $\sim 12^{\prime \prime}(9 \mathrm{kpc})$ at $1.5 \mathrm{GHz}$, the last two objects only extend for $\sim 2^{\prime \prime}$, well below the LOFAR resolution. All of them are relatively high power sources (between 0.3 and $1.0 \times 10^{23} \mathrm{~W} \mathrm{~Hz}^{-1}$ at $150 \mathrm{MHz}$ ) and with a spectral index $\alpha \sim 0.3$. Besides the four FR 0s with jetted morphology, the VLA images of Baldi et al. (2019) indicate that there are three further objects (out of 18) with a steep $(\alpha>0.5)$ spectrum between 1.4 and $4.5 \mathrm{GHz}$. These are unresolved objects, with sizes $\lesssim 00^{\prime \prime} 3(\lesssim 0.2 \mathrm{kpc})$, with a 


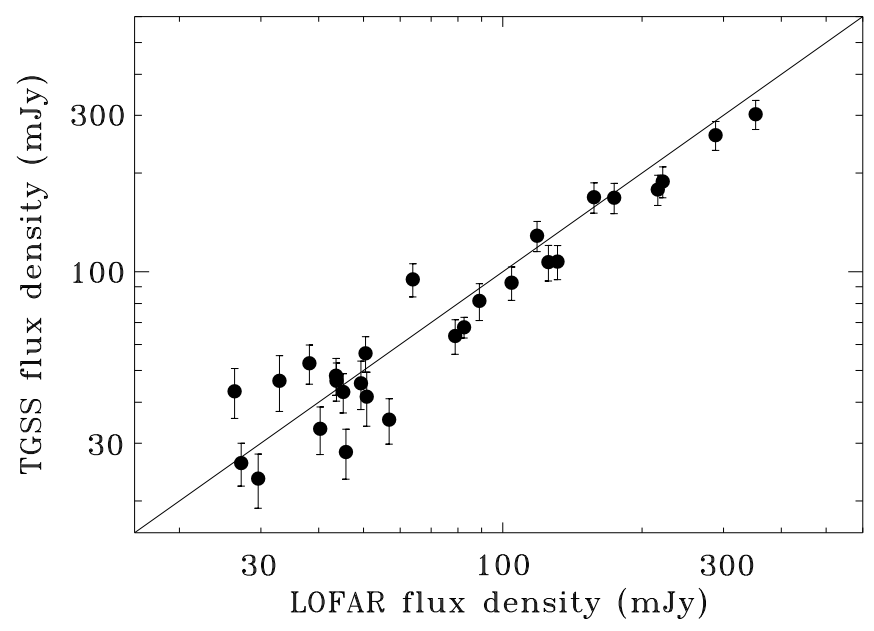

Fig. 6. Comparison of the flux densities of the 28 FR0CAT detected by TGSS and observed by LOFAR. The errors on the LOFAR fluxes, not reported, are dominated by the $\sim 10 \%$ uncertainty in the absolute calibration.

dominant contribution from the extended emission from their small scale jets.

The overall fraction of FR 0s showing evidence for the presence of jets can be estimated by including in the census those in which they are directly detected based on the available LOFAR, VLA, or VLBI (Cheng \& An 2018) images and those in which the spectral slope indicates a dominance of an optically thin component, that is, those with $\alpha>0.5$. By considering the overlap between these classes we obtain a lower limit to the fraction of jetted FR 0 of $\gtrsim 40 \%$.

The morphologies of the extended structures observed with LOFAR in the FR 0s recall what has been recently observed in other radio galaxies and in particular in the radio source associated with NGC 3998 (Sridhar et al. 2020). This galaxy produces a low-power and highly core dominated source with two elongated and distorted lobes of low-surface brightness with a total size of $\sim 30 \mathrm{kpc}$. The overall properties of NGC 3998 are consistent with a FR 0 classification, very similar to the FR0CAT sources with extended structures we found in this study. The spectral slope between $150 \mathrm{MHz}$ and $1.4 \mathrm{GHz}$ of the extended emission in NGC 3998 is $\alpha \sim 0.6$ suggesting that it is still actively fed by the AGN and it is not a relic structure. It would be clearly very important to measure the spectral indices of the extended emission in the FR 0s to test our suggestion that they are sources actively fed by their jets, but the depth of the available higher frequency images is not sufficient to provide strong constraints.

Other classes of AGN show radio structures reminiscent of those seen in FR 0s. In particular it has long been known that Seyfert galaxies, despite their definition as radio-quiet objects, often show extended radio emission (for example, Ulvestad \& Wilson 1984; Nagar et al. 2002, 2005) extending over a few kpc and with a high core dominance. More recently, Baldi et al. (2018b) found that most nearby LINERs are radio emitters, albeit of very low-power (typically $\sim 10^{20} \mathrm{~W} \mathrm{~Hz}^{-1}$ ). They are often associated with radio-jets and are also highly core-dominated. LINERs share with the FR 0s the typical range of values of optical line ratios, used for the spectroscopic classification (see, Buttiglione et al. 2010). FR 0s, FR Is, and LINERs also have a similar ratio between the core and the optical line luminosity, an indication of a similar efficiency of the central engine to produce highly relativistic electrons. These similarities are particularly interesting considering that the hosts of these radio-quiet AGN include late-type galaxies and lowmass ellipticals.

\section{Summary and conclusions}

We explored the low-frequency radio properties of the sample of compact radio sources associated with nearby $(z<0.05)$ massive early-type galaxies, FR0CAT, by using LOFAR observations at $150 \mathrm{MHz}$, available for 66 out of 104 FR 0s: all of them are detected, usually showing point-like structures. Resolved radio emission of low surface brightness is detected in 12 FR 0s: it contributes from between $5 \%$ and $40 \%$ of the total radio power at $150 \mathrm{MHz}$. The LOFAR observations confirm the general paucity of large scale emission in FR 0s, as already indicated by the FIRST images used for the selection of the sample. The extended radio emission usually have a jetted morphology extending between 15 and $40 \mathrm{kpc}$. In the remaining FR 0 s we set an upper limit to any extended emission of $\lesssim 5 \times 10^{22} \mathrm{~W} \mathrm{~Hz}^{-1}$, a factor $10-10^{3}$ below the typical luminosity of FR Is. It is likely that the FR 0s in which we detected large scale emission are just the tip of the iceberg of a broad distribution of extended power.

The spectral slopes of FR 0 s between $150 \mathrm{MHz}$ and $1.4 \mathrm{GHz}$ span a broad range $(-0.7 \lesssim \alpha \lesssim 0.8$, median $\bar{\alpha} \sim 0.1) ; 20 \%$ of them have steep spectra $(\alpha \gtrsim 0.5)$, an indication of the presence of substantial extended emission confined within the spatial resolution limit $(\sim 3-6 \mathrm{kpc}$ at $z \sim 0.05)$. The fraction of FR 0s showing evidence for the presence of jets, by including both spectral and morphological information, is at least $\sim 40 \%$.

Our study confirms that FR 0 s and FR I can be interpreted as two extremes of a continuous population of radio sources characterized by a broad distribution of sizes and luminosities of their extended radio emission, from low-luminosity compact RGs to Mpc-scale FRI s (see, Mingo et al. 2019). In this context, the widespread presence of jets in FR 0 s, an indication derived in a substantial fraction of the FR0CAT sample, either from the morphology of their resolved structures or from their spectral shape, is of great importance. FR 0s thus represent the low-end in size and power of jetted radio sources. The properties of these sources confirm the continuity of the properties of low-power radio galaxies starting from the compact FR 0s, characterized by a paucity of extended emission, small linear sizes, and high values of core dominance, to the most powerful FR Is which can extend for hundreds of kpc. Most likely, these differences are driven by the different properties of their jets, being lighter and/or slower in FR 0s, leading to a lower momentum flux. For this reason they are more subject to the effects of instabilities, turbulence, and entrainment causing their premature disruption and limiting their expansion to subgalactic scales.

Alternatively, FR0s might experience recurrent short-phases of activity. Large scale radio structures are preferentially seen in FR 0s whose host galaxies are more luminous and are located in a denser environment than average. The role of host's mass and environment in determining the properties of a radio source are unclear, but these results suggest the presence of a connection between these two parameters with the jet power. For example, short activity phases might be more commonly triggered in a poor group environment due to the smaller amount hot gas available in comparison with cluster of galaxies.

Various questions about the FR Os remain open and require further studies. The fraction of young radio galaxies among the FR Os is not well constrained. Such objects must exist as they represent the early phase of the evolution of the extended radio sources and carry important information about the onset 
of nuclear activity. Young radio sources (including, for example, compact steep and giga-hertz peaked sources) are characterized by high curvature convex spectra, with a low frequency turn-over due to absorption. While we have now adequate low frequency data, the overall spectral shape of FR 0s can not be properly studied due to the lack of sufficient information at higher frequencies. Similarly, higher resolution observations are required to detect and isolate the jet emission and to compare their properties (measuring the jets asymmetry, related to their speed) with those of the FR Is. The LOFAR international baselines will play a key role in this research, because at $150 \mathrm{MHz}$ the contrast between the steep jets and the flat cores is enhanced with respect to higher frequencies. Finally, deep observations at a resolution similar to that obtained with LOFAR are needed for a measurement of the spectral indices of the extended emission of FR 0s and to discriminate between sources actively fed by their jets and relic emission.

Acknowledgements. MB acknowledges support from the ERC-Stg DRANOEL, no. 714245. MJH acknowledges support from the UK Science and Technology Facilities Council (ST/R000905/1). HR acknowledges support from the ERC Advanced Investigator programme NewClusters 321271. PNB is grateful for support from the UK STFC via grant ST/R000972/1. This paper is based on data obtained from the International LOFAR Telescope (ILT). LOFAR (van Haarlem et al. 2013) is the Low-Frequency Array designed and constructed by ASTRON. It has observing, data processing, and data storage facilities in several countries, which are owned by various parties (each with their own funding sources), and are collectively operated by the ILT foundation under a joint scientific policy. The ILT resources have benefitted from the following recent major funding sources: CNRS-INSU, Observatoire de Paris and Université d'Orléans, France; BMBF, MIWF-NRW, MPG, Germany; Science Foundation Ireland (SFI), Department of Business, Enterprise and Innovation (DBEI) Ireland; NWO, The Netherlands; The Science and Technology Facilities Council, UK; Ministry of Science and Higher Education, Poland; Istituto Nazionale di Astrofisica (INAF), Italy. This research made use of the Dutch national e-infrastructure with support of the SURF Cooperative (e-infra 180169) and the LOFAR e-infra group. The Jülich LOFAR Long Term Archive and the German LOFAR network are both coordinated and operated by the Jülich Supercomputing Centre (JSC), and computing resources on the Supercomputer JUWELS at JSC were provided by the Gauss Centre for Supercomputing e.V. (Grant CHTB00) through the John von Neumann Institute for Computing (NIC). This research made use of the University of Hertfordshire high-performance computing facility and the LOFAR-UK computing facility located at the University of Hertfordshire and supported by STFC [ST/P000096/1], and of the LOFAR IT computing infrastructure supported and operated by INAF, and by the Physics
Dept. of Turin University (under the agreement with Consorzio Interuniversitario per la Fisica Spaziale) at the C3S Supercomputing Centre, Italy.

\section{References}

Baldi, R. D., \& Capetti, A. 2009, A\&A, 508, 603

Baldi, R. D., Capetti, A., \& Giovannini, G. 2015, A\&A, 576, A38

Baldi, R. D., Capetti, A., \& Massaro, F. 2018a, A\&A, 609, A1

Baldi, R. D., Williams, D. R. A., McHardy, I. M., et al. 2018b, MNRAS, 476, 3478

Baldi, R. D., Capetti, A., \& Giovannini, G. 2019, MNRAS, 482, 2294

Becker, R. H., White, R. L., \& Helfand, D. J. 1995, ApJ, 450, 559

Best, P. N., \& Heckman, T. M. 2012, MNRAS, 421, 1569

Brienza, M., Godfrey, L., Morganti, R., et al. 2016, A\&A, 585, A29

Buttiglione, S., Capetti, A., Celotti, A., et al. 2010, A\&A, 509, A6

Capetti, A., Massaro, F., \& Baldi, R. D. 2017, A\&A, 598, A49

Capetti, A., Baldi, R. D., Brienza, M., Morganti, R., \& Giovannini, G. 2019, A\&A, 631, A176

Capetti, A., Massaro, F., \& Baldi, R. D. 2020, A\&A, 633, A161

Cheng, X.-P., \& An, T. 2018, ApJ, 863, 155

Condon, J. J., Cotton, W. D., Greisen, E. W., et al. 1998, AJ, 115, 1693

Fanaroff, B. L., \& Riley, J. M. 1974, MNRAS, 167, 31P

Garofalo, D., \& Singh, C. B. 2019, ApJ, 871, 259

Ghisellini, G. 2011, in American Institute of Physics Conference Series, eds. F. A. Aharonian, W. Hofmann, \& F. M. Rieger, AIP Conf. Ser., 1381, 180

Hardcastle, M. J., Alexander, P., Pooley, G. G., \& Riley, J. M. 1998, MNRAS, 296, 445

Hardcastle, M. J., Gürkan, G., van Weeren, R. J., et al. 2016, MNRAS, 462, 1910

Helfand, D. J., White, R. L., \& Becker, R. H. 2015, ApJ, 801, 26

Intema, H. T., Jagannathan, P., Mooley, K. P., \& Frail, D. A. 2017, A\&A, 598, A78

Longair, M. S. 1994, High Energy Astrophysics (Cambridge University Press)

Mingo, B., Croston, J. H., Hardcastle, M. J., et al. 2019, MNRAS, 488, 2701

Nagar, N. M., Falcke, H., Wilson, A. S., \& Ulvestad, J. S. 2002, A\&A, 392, 53

Nagar, N. M., Falcke, H., \& Wilson, A. S. 2005, A\&A, 435, 521

Sadler, E. M., Ekers, R. D., Mahony, E. K., Mauch, T., \& Murphy, T. 2014, MNRAS, 438, 796

Shimwell, T. W., Röttgering, H. J. A., Best, P. N., et al. 2017, A\&A, 598, A104

Shimwell, T. W., Tasse, C., Hardcastle, M. J., et al. 2019, A\&A, 622, A1

Sridhar, S. S., Morganti, R., Nyland, K., et al. 2020, A\&A, 634, A108

Swarup, G. 1991, in IAU Colloq. 131: Radio Interferometry. Theory, Techniques, and Applications, eds. T. J. Cornwell, \& R. A. Perley, ASP Conf. Ser., 19, 376

Torresi, E., Grandi, P., Capetti, A., Baldi, R. D., \& Giovannini, G. 2018, MNRAS, 476, 5535

Ulvestad, J. S., \& Wilson, A. S. 1984, ApJ, 278, 544

van Haarlem, M. P., Wise, M. W., Gunst, A. W., et al. 2013, A\&A, 556, A2

York, D. G., Adelman, J., Anderson, J. E., Jr, et al. 2000, AJ, 120, 1579 
Appendix A: Radio properties of the sample

Table A.1. Radio properties of the sample.

\begin{tabular}{|c|c|c|c|c|c|c|c|c|c|c|}
\hline SDSS name & $z$ & $\mathrm{rms}$ & \multicolumn{2}{|c|}{$\begin{array}{c}\mathrm{F}(150) \\
\text { Central Total } \\
\end{array}$} & Size & Morph. & $\mathrm{L}(150)$ & $\mathrm{F}(1.4)$ & $\alpha$ & Im. type \\
\hline J010852.48-003919.4 & 0.045 & 0.26 & 6.8 & & $3.9 \pm 0.8$ & & 22.52 & 13.0 & -0.29 & $\mathrm{P}$ \\
\hline J011204.61-001442.4 & 0.044 & 0.29 & 8.0 & & $2.5 \pm 0.3$ & & 22.57 & 18.9 & -0.38 & $\mathrm{P}$ \\
\hline J011515.78+001248.4 & 0.045 & 0.23 & 23.9 & & $4.0 \pm 0.3$ & & 23.06 & 45.0 & -0.28 & $\mathrm{P}$ \\
\hline J075354.98+130916.5 & 0.048 & 0.21 & 3.5 & & $7.5 \pm 1.7$ & & 22.28 & 12.6 & -0.58 & $\mathrm{P}$ \\
\hline J080716.58+145703.3 & 0.029 & 0.20 & 26.3 & 32.6 & $8.8 \pm 0.5$ & Core-jet & 22.72 & 25.5 & 0.01 & $\mathrm{P}$ \\
\hline $\mathrm{J} 083158.49+562052.3$ & 0.045 & 0.06 & 27.2 & & $3.2 \pm 0.1$ & & 23.12 & 9.5 & 0.47 & DR2 \\
\hline J084102.73+595610.5 & 0.038 & 0.06 & 15.2 & & $4.1 \pm 0.1$ & & 22.72 & 11.1 & 0.14 & DR2 \\
\hline J090652.79+412429.7 & 0.027 & 0.08 & 17.0 & & $4.4 \pm 0.1$ & & 22.47 & 58.2 & -0.55 & DR2 \\
\hline J090734.91+325722.9 & 0.049 & 0.09 & 81.3 & & $10.2 \pm 0.1$ & & 23.67 & 42.9 & 0.29 & DR2 \\
\hline J090937.44+192808.2 & 0.028 & 0.27 & 174.1 & & $3.9 \pm 0.2$ & & 23.51 & 68.6 & 0.42 & $\mathrm{P}$ \\
\hline J091039.92+184147.6 & 0.028 & 0.20 & 157.4 & 480.7 & $4.4 \pm 0.2$ & Diffuse & 23.47 & 47.4 & 0.54 & $\mathrm{P}$ \\
\hline J091601.78+173523.3 & 0.029 & 0.27 & 63.9 & 157.6 & $5.9 \pm 0.6$ & Head-tail & 23.11 & 17.3 & 0.58 & $\mathrm{P}$ \\
\hline J093003.56+341325.3 & 0.042 & 0.09 & 89.0 & & $5.0 \pm 0.1$ & & 23.58 & 30.8 & 0.48 & DR2 \\
\hline J093938.62+385358.6 & 0.046 & 0.06 & 4.3 & & $35.4 \pm 2.5$ & & 22.34 & 6.2 & -0.16 & DR2 \\
\hline J094319.15+361452.1 & 0.022 & 0.07 & 56.8 & & $5.7 \pm 0.1$ & & 22.82 & 74.9 & -0.12 & DR2 \\
\hline $\mathrm{J} 102403.28+420629.8$ & 0.044 & 0.06 & 5.0 & & $3.6 \pm 0.1$ & & 22.37 & 5.4 & -0.03 & $\mathrm{DR} 2$ \\
\hline $\mathrm{J} 102511.50+171519.9$ & 0.045 & 0.21 & 8.6 & 14.6 & $8.7 \pm 0.9$ & Two-sided & 22.62 & 9.9 & -0.06 & $\mathrm{P}$ \\
\hline $\mathrm{J} 103719.33+433515.3$ & 0.025 & 0.18 & 288.4 & & $4.8 \pm 0.1$ & & 23.64 & 128.9 & 0.36 & DR2 \\
\hline $\mathrm{J} 104403.68+435412.0$ & 0.025 & 0.09 & 29.6 & 43.5 & $15.9 \pm 0.1$ & Two-sided & 22.65 & 23.2 & 0.11 & DR2 \\
\hline $\mathrm{J} 104852.92+480314.8$ & 0.041 & 0.10 & 78.9 & & $5.0 \pm 0.1$ & & 23.50 & 18.9 & 0.64 & DR2 \\
\hline $\mathrm{J} 105731.16+405646.1$ & 0.025 & 0.08 & 40.3 & & $6.3 \pm 0.1$ & & 22.78 & 47.3 & -0.07 & DR2 \\
\hline $\mathrm{J} 111113.18+284147.0$ & 0.029 & 0.10 & 50.5 & & $3.1 \pm 0.1$ & & 23.01 & 43.6 & 0.07 & DR2 \\
\hline $\mathrm{J} 111622.70+291508.2$ & 0.045 & 0.20 & 10.2 & 16.5 & $4.7 \pm 0.3$ & Two-sided & 22.69 & 73.1 & -0.88 & DR2 \\
\hline $\mathrm{J} 111700.10+323550.9$ & 0.035 & 0.08 & 23.7 & & $5.0 \pm 0.1$ & & 22.84 & 19.2 & 0.09 & DR2 \\
\hline $\mathrm{J} 112256.47+340641.3$ & 0.043 & 0.07 & 43.7 & & $3.6 \pm 0.1$ & & 23.29 & 16.6 & 0.43 & $\mathrm{DR} 2$ \\
\hline $\mathrm{J} 112625.19+520503.5$ & 0.048 & 0.12 & 37.1 & & $5.0 \pm 0.1$ & & 23.31 & 7.8 & 0.70 & DR2 \\
\hline $\mathrm{J} 112727.52+400409.4$ & 0.035 & 0.09 & 45.2 & & $5.9 \pm 0.1$ & & 23.12 & 14.8 & 0.50 & DR2 \\
\hline $\mathrm{J} 113449.29+490439.4$ & 0.033 & 0.14 & 125.5 & 140.0 & $6.8 \pm 0.1$ & Two-sided & 23.51 & 25.1 & 0.72 & DR2 \\
\hline $\mathrm{J} 113637.14+510008.5$ & 0.050 & 0.10 & 2.0 & & $3.8 \pm 0.3$ & & 22.08 & 7.8 & -0.61 & DR2 \\
\hline J114232.84+262919.9 & 0.030 & 0.11 & 8.6 & & $3.3 \pm 0.2$ & & 22.27 & 44.7 & -0.74 & $\mathrm{P}$ \\
\hline $\mathrm{J} 114804.60+372638.0$ & 0.042 & 0.06 & 5.3 & & $5.0 \pm 0.2$ & & 22.35 & 29.2 & -0.76 & DR2 \\
\hline $\mathrm{J} 115531.39+545200.4$ & 0.050 & 0.33 & 21.0 & & $2.5 \pm 0.2$ & & 23.10 & 31.8 & -0.19 & DR2 \\
\hline $\mathrm{J} 120551.46+203119.0$ & 0.024 & 0.21 & 104.5 & & $2.9 \pm 0.2$ & & 23.16 & 102.7 & 0.01 & $\mathrm{P}$ \\
\hline $\mathrm{J} 120607.81+400902.6$ & 0.037 & 0.05 & 8.0 & & $2.8 \pm 0.1$ & & 22.42 & 8.9 & -0.05 & DR2 \\
\hline $\mathrm{J} 121329.27+504429.4$ & 0.031 & 0.27 & 216.3 & & $6.4 \pm 0.1$ & & 23.70 & 102.7 & 0.33 & DR2 \\
\hline $\mathrm{J} 121951.65+282521.3$ & 0.027 & 0.10 & 7.6 & & $8.0 \pm 0.4$ & & 22.12 & 8.0 & -0.02 & DR2 \\
\hline $\mathrm{J} 122421.31+600641.2$ & 0.044 & 0.08 & 16.0 & & $5.4 \pm 0.1$ & & 22.87 & 5.7 & 0.46 & DR2 \\
\hline $\mathrm{J} 123011.85+470022.7$ & 0.039 & 0.16 & 118.6 & & $3.3 \pm 0.1$ & & 23.64 & 87.5 & 0.14 & DR2 \\
\hline $\mathrm{J} 130837.91+434415.1$ & 0.036 & 0.14 & 131.3 & & $5.1 \pm 0.1$ & & 23.61 & 52.3 & 0.41 & DR2 \\
\hline $\mathrm{J} 133042.51+323249.0$ & 0.034 & 0.09 & 19.9 & & $4.1 \pm 0.1$ & & 22.74 & 16.9 & 0.07 & DR2 \\
\hline $\mathrm{J} 133737.49+155820.0$ & 0.026 & 0.76 & 50.8 & & $7.7 \pm 0.4$ & & 22.92 & 30.1 & 0.23 & $\mathrm{P}$ \\
\hline $\mathrm{J} 134159.72+294653.5$ & 0.045 & 0.09 & 14.9 & & $4.9 \pm 0.1$ & & 22.86 & 9.4 & 0.21 & DR2 \\
\hline $\mathrm{J} 135036.01+334217.3$ & 0.014 & 0.09 & 352.0 & & $3.9 \pm 0.1$ & & 23.22 & 99.7 & 0.56 & DR2 \\
\hline $\mathrm{J} 140528.32+304602.0$ & 0.025 & 0.08 & 2.4 & & $5.0 \pm 0.5$ & & 21.56 & 7.6 & -0.52 & DR2 \\
\hline $\mathrm{J} 142724.23+372817.0$ & 0.032 & 0.08 & 52.7 & & $7.2 \pm 0.1$ & & 23.11 & 10.8 & 0.71 & DR2 \\
\hline $\mathrm{J} 143312.96+525747.3$ & 0.047 & 0.09 & 8.6 & & $5.1 \pm 0.1$ & & 22.66 & 15.2 & -0.25 & DR2 \\
\hline $\mathrm{J} 143424.79+024756.2$ & 0.028 & 0.36 & 6.3 & & $5.5 \pm 1.2$ & & 22.07 & 9.0 & -0.16 & $\mathrm{P}$ \\
\hline $\mathrm{J} 143620.38+051951.5$ & 0.029 & 0.48 & 15.6 & & $6.7 \pm 0.8$ & & 22.50 & 26.7 & -0.24 & $\mathrm{P}$ \\
\hline $\mathrm{J} 152010.94+254319.3$ & 0.034 & 0.16 & 10.7 & & $5.6 \pm 0.5$ & & 22.47 & 17.1 & -0.21 & $\mathrm{P}$ \\
\hline
\end{tabular}

Column description: (1) name; (2) redshift; (3) rms of the LOFAR images in mJy beam ${ }^{-1}$; (4) flux densities (in mJy) at $150 \mathrm{MHz}$ of the central radio component. Errors are dominated by the $\sim 10 \%$ flux scale uncertainty; (5) total $150 \mathrm{MHz}$ flux density (in mJy) for the extended sources; (6) deconvolved major axis of the central source in arcseconds; (7) morphological description of the extended emission, when present; (8) logarithm of the luminosity (in $\mathrm{W} \mathrm{Hz}^{-1}$ ) of the central component at $150 \mathrm{MHz}$; (9) flux density (in mJy) at $1.4 \mathrm{GHz}$ from FIRST; (10) spectral index $\alpha$ between $150 \mathrm{MHz}$ and $1.4 \mathrm{GHz}$ defined as $F_{v} \propto v^{-\alpha}$; errors for $\alpha$ are between 0.05 and 0.12 ; (11) image type: "DR2" = image in the DR2 area, "P" = individual LoTSS pointing with offset $<3^{\circ}$. 
Table A.1. continued

\begin{tabular}{lcccccccccc}
\hline \hline SDSS name & $z$ & rms & $\begin{array}{c}F(150) \\
\text { Central Total }\end{array}$ & Size & Morph. & L(150) & F(1.4) & $\alpha$ & Im. type \\
\hline J152151.85+074231.7 & 0.044 & 0.64 & 31.4 & $6.0 \pm 0.2$ & & 23.16 & 7.7 & 0.63 & P \\
J153016.15+270551.0 & 0.033 & 0.15 & 18.9 & & $3.5 \pm 0.3$ & & 22.69 & 13.4 & 0.15 & P \\
J154147.28+453321.7 & 0.037 & 0.10 & 14.8 & 30.9 & $9.0 \pm 0.1$ & Head-tail & 22.69 & 6.2 & 0.39 & DR2 \\
J154426.93+470024.2 & 0.038 & 0.09 & 32.9 & & $2.2 \pm 0.1$ & & 23.06 & 17.4 & 0.29 & DR2 \\
J154451.23+433050.6 & 0.037 & 0.15 & 3.2 & & $5.3 \pm 0.3$ & & 22.02 & 12.5 & -0.61 & DR2 \\
J155951.61+255626.3 & 0.045 & 0.62 & 82.5 & & $6.9 \pm 0.4$ & & 23.60 & 29.2 & 0.46 & P \\
J155953.99+444232.4 & 0.042 & 0.12 & 221.7 & & $5.1 \pm 0.1$ & & 23.97 & 58.8 & 0.59 & DR2 \\
J160426.51+174431.1 & 0.041 & 0.38 & 49.4 & 110.9 & $7.7 \pm 0.7$ & Head-tail & 23.30 & 71.8 & -0.17 & P \\
J160523.84+143851.6 & 0.041 & 0.21 & 24.4 & 26.4 & $10.4 \pm 0.5$ & Core-jet & 22.99 & 7.5 & 0.53 & P \\
J160641.83+084436.8 & 0.047 & 0.29 & 9.4 & & $6.3 \pm 0.6$ & & 22.69 & 8.8 & 0.03 & P \\
J161238.84+293836.9 & 0.032 & 0.09 & 5.8 & & $3.8 \pm 0.2$ & & 22.15 & 22.8 & -0.61 & DR2 \\
J161256.85+095201.5 & 0.017 & 0.33 & 38.2 & & $7.3 \pm 0.6$ & & 22.42 & 21.3 & 0.26 & P \\
J162944.98+404841.6 & 0.029 & 0.07 & 14.4 & & $6.2 \pm 0.1$ & & 22.46 & 6.9 & 0.33 & DR2 \\
J164925.86+360321.3 & 0.032 & 0.08 & 42.6 & & $5.0 \pm 0.1$ & & 23.02 & 10.5 & 0.63 & DR2 \\
J165830.05+252324.9 & 0.033 & 0.22 & 4.5 & & $7.2 \pm 0.7$ & & 22.07 & 14.8 & -0.53 & P \\
J170358.49+241039.5 & 0.031 & 0.22 & 45.8 & 58.7 & $12.5 \pm 0.7$ & Diffuse & 23.02 & 22.8 & 0.31 & P \\
J172215.41+304239.8 & 0.046 & 0.10 & 43.6 & 63.2 & $24.0 \pm 0.2$ & Two-sided & 23.34 & 5.7 & 0.91 & DR2 \\
\hline
\end{tabular}

